\title{
ATIVIDADE ELETRENCEFALOGRÁFICA PAROXÍSTICA NO PRIMEIRO ANO DE VIDA DE PACIENTES EPILÉTICOS
}

\author{
RUbens Moura Ribeiro
}

A atividade convulsiógena paroxística deve ser considerada como anormal em qualquer idade, e representa uma reação cerebral funcional, de tipo irritativo ${ }^{15}$. Entretanto, a interpretação do seu significado varia, conforme o autor considerado e o critério adotado. Assim, quando se considera a incidência dêsses achados no eletrencefalograma (EEG) de crianças, os estudos sistematizados revelam resultados contraditórios, decorrentes de modificações complexas que ocorrem durante a maturação cerebral 13, 16, 22, 29, 34 .

Entretanto, quando em 1966 analisamos os achados eletrencefalográficos de crianças com crises convulsivas, que ocorreram nos primeiros 12 meses de idade, chamou-nos a atenção a elevada incidência de respostas paroxísticas e a impossibilidade em se determinar até que ponto essas manifestações bioelétricas corresponderiam à presença de um mesmo substrato fisiopatológico ${ }^{34}$.

Essas alterações eletrencefalográficas, principalmente as de tipo irritativo, representam distúrbio funcional ou anatômico do encéfalo, e realçam a importância do conhecimento dos mecanismos fisiopatológicos ligados à origem e à propagação da descarga, para a compreensão das manifestações neurológicas e sua correlação com as alterações bioelétricas do cérebro de crianças epiléticas. O diagnóstico eletrencefalográfico pode fornecer uma idéia objetiva sôbre o significado de lesão cerebral com manifestações paroxísticas, pela correlação das alterações registradas nos traçados, com a síndrome clínica evidenciada no paciente. A interpretação correta do potencial patológico, nessas circunstâncias, é de grande importância prática no campo da eletrencefalografia clinica ${ }^{27}$.

Com o objetivo de investigar os aspectos qualitativos e quantitativos dessas manifestações eletrencefalográficas, estudamos um grupo de crianças com epilepsia, utilizando o EEG como método capaz de demonstrar o grau de comprometimento biolétrico durante um período limitado da maturação cerebral.

Resumo da tese apresentada para concurso à Docência Livre de Clinica Neurológica na Faculdade de Medicina de Ribeirão Prêto.

Nota do autor - Agradecemos ao Prof. Armbrust-Figueiredo e Assistentes da Clínica Neurológica da Faculdade de Medicina de Ribeirão Prêto, a orientação e apoio ao nosso trabalho. 


\section{MATERIAL E METTODOS}

O material é constituído de 80 crianças com epilepsia, selecionadas, dentre os pacientes examinados no Serviço de Eletrencefalografia do Departamento de Neurologia da Faculdade de Medicina de Ribeirão Prêto. Com o EEG procuramos determinar a incidência e distribuição topográfica da atividade paroxística cerebral registrada na vigília ou no sono.

Os pacientes foram examinados sob o aspecto neurológico e a anamnese focalizou particularmente as características da sintomatologia epilética, com intúito de se obter informações precisas sôbre os aspectos clínicos da crise. Dos 80 pacientes, 48 eram do sexo masculino e 32 do feminino; suas idades variaram entre dois dias e 12 meses. A tabela 1 apresenta os dados de identificação dos pacientes e o diagnóstico clínico de cada caso.

Os pacientes foram submetidos ao exame eletrencefalográfico, utilizando-se um aparelho Grass, com 8 canais. Na obtenção dos traçados utilizamos derivações bipolares e monopolares, segundo a técnica recomendada pela "International Federation for Electroencephalography and Clinical Neurophysiology" 42.

Em 6 pacientes o registro dos traçados foi realizado em estado de vigília; em 10, durante o sono espontâneo; em 61, durante o sono induzido por barbitúrico (Seconal), administrado por via oral, na dose de 5 a $10 \mathrm{mg}$ por $\mathrm{kg} /$ pêso; em três. o EEG foi realizado em vigilia e durante o sono, sendo que, em dois, durante sono espontâneo e, em um, durante o sono barbitúrico.

A interpretação dos traçados de vigília foi baseada nas informações fornecidas pelo Atlas de Eletrencefalografia de Gibbs e Bibbs ${ }^{15}$, e na caracterização dos padrões eletrencefalográficos do sono, consideramos sòmente os eventos manifestados nas fases do sono com atividade lenta, segundo o critério estabelecido inicialmente por Delange e col. ${ }^{6}$, depois modificado por Hess ${ }^{18}$.

$\mathrm{Na}$ sistematização das síndromes epileticas, foram considerados os critérios estabelecidos pela "International League Against Epilepsy" 11.

\section{R E S U L T A D O S}

A análise de nossos resultados evidenciou que 70 pacientes $(87,5 \%)$ apresentavam EEG alterado e $10(12,5 \%)$ apresentavam EEG normal.

As alterações foram caracterizadas pela presença de atividade paroxística, registrada 77 vêzes, e por padrōes bioelétricos não paroxísticos, registrados 12 vêzes.

A incidência dos diferentes padrões bioelétricos paroxísticos, não paroxísticos e normais, registrados no EEG realizado durante o sono ou na vigília, está representada na tabela 2.

A relação entre os diferentes tipos de alteraçōes paroxísticas e o número de pacientes está assinalada na tabela 3 .

Os diferentes tipos de alterações paroxísticas, ou não, de projeção unilateral e bilateral estão representados na tabela 4 .

Os casos com associação de duas ou mais alterações eletrencefalográficas foram analisados na tabela 5 .

A distribuição das diversas alterações paroxísticas, ou não, de acôrdo com sua topografia em cada área cerebral, está registrada na tabela 6.

A correlação entre os diferentes padrões bioelétricos paroxísticos, ou não, e as crises clínicas está representada na tabela 7 . 


\begin{tabular}{|c|c|c|c|c|c|}
\hline N.o & Nome & EEG & Sexo & Idade & Clínica \\
\hline 1 & MG & 6748 & $\mathbf{F}$ & 29 & Bráculo-facial $\mathbf{E}$ \\
\hline$\frac{1}{2}$ & LESR & 4305 & $\mathbf{M}$ & $5 \mathrm{~d}$ & Generalizada \\
\hline 3 & HS & 4942 & $\mathbf{F}$ & $7 \mathrm{~d}$. & Bráquio-facial E \\
\hline 4 & ASM & 3125 & $\mathrm{~F}$ & $8 \mathrm{~d}$ & Hemicorpo D \\
\hline 5 & LR & 5927 & $\mathbf{F}$ & $19 \mathrm{~d}$. & Generalizada \\
\hline 6 & SHL & 1425 & $\mathbf{F}$ & $26 \mathrm{~d}$. & Hemicorpo D \\
\hline 7 & MFS & 1958 & $\mathrm{~F}$ & $29 \mathrm{~d}$. & Bráquio-facial D \\
\hline 8 & CRM & 3541 & $\mathrm{~F}$ & $30 \mathrm{~d}$. & Bráquio-facial D \\
\hline 9 & DAS & 6555 & $\mathbf{M}$ & $30 \mathrm{~d}$. & Bráquio-facial D \\
\hline 10 & MCC & 3875 & $\mathbf{M}$ & $30 \mathrm{~d}$. & Bráquio-facial E \\
\hline 11 & MOI & 5861 & $\mathbf{M}$ & $50 \mathrm{~d}$ & Generalizada \\
\hline 12 & $\mathrm{ACP}$ & 5041 & $\mathbf{F}$ & $2 \mathrm{~m}$. & Ausência \\
\hline$\overline{13}$ & JG & 3395 & $\mathbf{F}$ & $2 \mathrm{~m}$. & Generalizada \\
\hline 14 & PRS & 3183 & $\mathbf{M}$ & $3 \mathrm{~m}$ & Ausência \\
\hline 15 & VS & 3670 & $\mathbf{F}$ & $3 \mathrm{~m}$. & Tônico postural \\
\hline 16 & ZIM & 4277 & $\mathbf{F}$ & $3 \mathrm{~m}$. & Generalìzada \\
\hline 17 & MAT & 4552 & $\mathbf{M}$ & $3 \mathrm{~m}$. & Ausência \\
\hline 18 & VFD & 5693 & $\mathbf{F}$ & $3 \mathrm{~m}$ & Espasmo em flexão \\
\hline 19 & AFR & 5769 & $\mathrm{M}$ & $3 \mathrm{~m}$. & Hemicorpo D \\
\hline 20 & MTG & 1713 & $\mathbf{F}$ & $4 \mathrm{~m}$ & Atônica \\
\hline 21 & RBB & 2045 & $\mathbf{M}$ & $4 \mathrm{~m}$ & Hemicorpo $\mathrm{D}$ \\
\hline 22 & SV & $\mathbf{3 3 7 4}$ & $\mathrm{F}$ & $4 \mathrm{~m}$ & Bráqu:o-facial D \\
\hline 23 & SHBM & 1601 & $\mathbf{F}$ & $5 \mathrm{~m}$. & Espasmo em flexão \\
\hline 24 & AUA & 2273 & $\mathrm{M}$ & $5 \mathrm{~m}$ & Generalizada \\
\hline $\begin{array}{l}24 \\
25\end{array}$ & JAP & 3396 & M & $5 \mathrm{~m}$ & Hemicorpo D \\
\hline 26 & MAT & 3588 & M & $5 \mathrm{~m}$. & Generalizada \\
\hline 27 & CAM & 4564 & $\mathrm{M}$ & $5 \mathrm{~m}$. & Espasmo em flexão \\
\hline 28 & $\mathrm{AMC}$ & 5618 & M & $5 \mathrm{~m}$. & Espasmo em flexão \\
\hline 29 & VRF & 4730 & $\mathrm{~F}$ & $5 \mathrm{~m}$. & Generalizada \\
\hline 30 & RMS & 5638 & $\mathrm{~F}$ & $5 \mathrm{~m}$. & Hemicorpo D \\
\hline 31 & MG & 5899 & M & $5 \mathrm{~m}$. & Espasmo em flexão \\
\hline 32 & JVD & 760 & $\mathbf{F}$ & $6 \mathrm{~m}$ & Bráquio-facial E \\
\hline 33 & $\mathrm{CMC}$ & 1732 & $\mathrm{M}$ & $6 \mathrm{~m}$. & Espasmo em flexão \\
\hline 34 & AMAP & 1953 & $\mathbf{M}$ & $6 \mathrm{~m}$. & Generalizada \\
\hline 35 & JAP & 3469 & $\mathbf{M}$ & $6 \mathrm{~m}$ & Hemicorpo D \\
\hline 36 & MB & 4214 & $\mathbf{F}$ & $6 \mathrm{~m}$ & Tônico postural \\
\hline 37 & MRJ & 4709 & $\mathbf{M}$ & $6 \mathrm{~m}$ & Atônica \\
\hline 38 & MJS & 4768 & M & $6 \mathrm{~m}$ & Generalizada \\
\hline 39 & ECS & 4134 & $\mathbf{F}$ & $7 \mathrm{~m}$ & Generalizada \\
\hline 40 & SAS & 4848 & $\mathrm{M}$ & $7 \mathrm{~m}$ & Bráquio-facial E \\
\hline 41 & MEA & 5553 & $\mathbf{M}$ & $7 \mathrm{~m}$. & Hemicorpo D \\
\hline 42 & SII & 6831 & $\mathbf{M}$ & $7 \mathrm{~m}$ & Ausência \\
\hline 43 & SM & 1533 & $\mathbf{F}$ & $8 \mathrm{~m}$. & Hemicorpo $\mathrm{E}$ \\
\hline 44 & LSS & 2480 & $\mathbf{M}$ & $8 \mathrm{~m}$ & Generalizada \\
\hline 45 & CAPL & 3251 & M & $8 \mathrm{~m}$. & Espasmo em flexão \\
\hline 46 & EDF & 4025 & $\mathbf{M}$ & $8 \mathrm{~m}$. & Ausência \\
\hline 47 & SMB & 4291 & $\mathrm{~F}$ & $8 \mathrm{~m}$. & Generalizada \\
\hline 48 & EMR & 4551 & $\mathrm{~F}$ & $8 \mathrm{~m}$. & Ausência \\
\hline 49 & JPS & 4589 & $\mathrm{M}$ & $8 \mathrm{~m}$. & Hemicorpo $\mathrm{E}$ \\
\hline 50 & JLP & 5055 & M & $8 \mathrm{~m}$. & Hemicorpo D \\
\hline 51 & MAC & 6007 & $\mathbf{F}$ & $8 \mathrm{~m}$. & Tônico postural \\
\hline 52 & CM & 6300 & $\mathbf{F}$ & $8 \mathrm{~m}$. & Atônica \\
\hline 53 & $\mathrm{ER}$ & 6539 & $\mathbf{M}$ & $8 \mathrm{~m}$. & Espasmo em flexão \\
\hline 54 & VL & 6615 & $\mathbf{F}$ & $8 \mathrm{~m}$ & Generalizada \\
\hline 55 & ECA & 6901 & $\mathbf{M}$ & $8 \mathrm{~m}$. & Generalizada \\
\hline 56 & RO & 1721 & $\mathrm{~F}$ & $9 \mathrm{~m}$ & Hemicorpo $\mathrm{E}$ \\
\hline 57 & JRI & 4402 & M & $9 \mathrm{~m}$. & Espasmo em flexäo \\
\hline 58 & JASS & 4906 & M & $9 \mathrm{~m}$ & Tônico postural \\
\hline 59 & DS & 6136 & $\mathbf{M}$ & $9 \mathrm{~m}$. & Espasmo em flexão \\
\hline 60 & CJC & 4229 & $\mathbf{M}$ & $10 \mathrm{~m}$. & Espasmo em flexão \\
\hline 61 & AJM & 4441 & M & $10 \mathrm{~m}$. & Generalizada \\
\hline 62 & $\mathrm{ACB}$ & 6375 & $\mathrm{M}$ & $10 \mathrm{~m}$. & Ausência \\
\hline 63 & $\mathrm{AB}$ & 2301 & M & $11 \mathrm{~m}$. & Generalizada \\
\hline 64 & FCGD & 4690 & $\mathrm{M}$ & $11 \mathrm{~m}$. & Ausência \\
\hline 65 & $\mathrm{ACC}$ & 1182 & $\mathrm{M}$ & $12 \mathrm{~m}$. & Hemicorpo D \\
\hline 66 & $\mathrm{RB}$ & 2348 & $\mathrm{M}$ & $12 \mathrm{~m}$. & Hemicorpo D \\
\hline 67 & $\mathrm{CN}$ & 2412 & $\mathbf{F}$ & $12 \mathrm{~m}$. & Hemicorpo D \\
\hline 68 & PCCC & 3010 & $\mathbf{F}$ & $12 \mathrm{~m}$. & Ausência \\
\hline 69 & TVHS & 3550 & $\mathbf{F}$ & $12 \mathrm{~m}$. & Hemicorpo $\mathrm{E}$ \\
\hline 70 & MLR & 3926 & $\mathbf{F}$ & $12 \mathrm{~m}$. & Bráquio-facial E \\
\hline 71 & JLY & 4142 & $\mathrm{M}$ & $12 \mathrm{~m}$. & Tônico postural \\
\hline 72 & $\mathrm{RH}$ & 4197 & $\mathbf{M}$ & $12 \mathrm{~m}$. & Generalizada \\
\hline 73 & AS & 4236 & $\mathrm{M}$ & $12 \mathrm{~m}$. & Generalizada \\
\hline 74 & GO & 4434 & M & $12 \mathrm{~m}$. & Bráquio-facial D \\
\hline 75 & LBP & 4456 & M & $12 \mathrm{~m}$. & Generalizada \\
\hline 76 & FRV & 4568 & $\mathbf{F}$ & $12 \mathrm{~m}$. & Generalizada \\
\hline 77 & ECSM & 4594 & $\mathrm{M}$ & $12 \mathrm{~m}$. & Generalizada \\
\hline 78 & $\mathrm{CM}$ & $\mathbf{5 1 0 0}$ & $\mathrm{M}$ & $12 \mathrm{~m}$. & Hemicorpo D \\
\hline 79 & MS & 5692 & M & $12 \mathrm{~m}$. & Hemicorpo $\mathrm{E}$ \\
\hline 80 & VV & 5981 & $\mathrm{M}$ & $12 \mathrm{~m}$. & Hemicorpo D \\
\hline
\end{tabular}




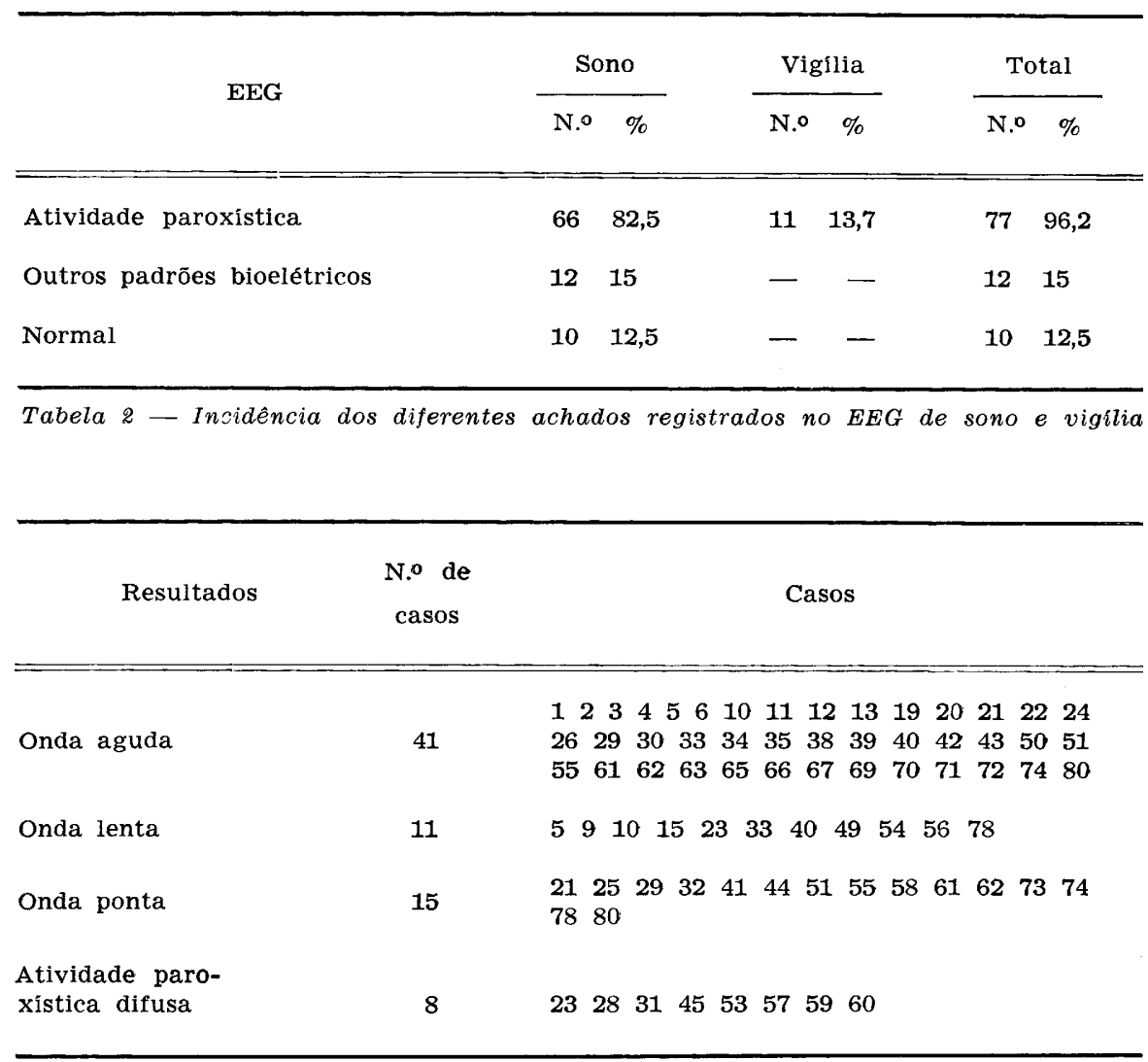

Tabela 3 - Incidência das alterações eletrencefalográficas paroxísticas

\begin{tabular}{|c|c|c|c|c|c|c|c|c|}
\hline \multirow[t]{2}{*}{ Tipos de alteração } & \multicolumn{2}{|c|}{$\begin{array}{c}\text { Alteraçōes } \\
\text { focais }\end{array}$} & \multicolumn{2}{|c|}{$\begin{array}{c}\text { unilaterais } \\
\text { difusas }\end{array}$} & \multicolumn{2}{|c|}{$\begin{array}{l}\text { Alterações } \\
\text { focais }\end{array}$} & \multicolumn{2}{|c|}{$\begin{array}{r}\text { bilaterais } \\
\text { difusas }\end{array}$} \\
\hline & N.o & $\%$ & N.o & $\%$ & N.o & $\%$ & N.o & $\%$ \\
\hline Onda aguda & 29 & 36,2 & 10 & 12,5 & 2 & 2,5 & 一 & $\longrightarrow$ \\
\hline Onda lenta & 7 & 8,7 & 3 & 3,7 & 1 & 1,2 & 一 & $\longrightarrow$ \\
\hline Onda ponta & 11 & 13,7 & 4 & 5 & - & - & $\longrightarrow$ & 一 \\
\hline Atividade paroxística difusa & 一 & - & - & - & - & - & 8 & 10 \\
\hline Atividade não paroxística & 2 & 2,5 & 4 & 5 & - & - & 6 & 7,5 \\
\hline
\end{tabular}




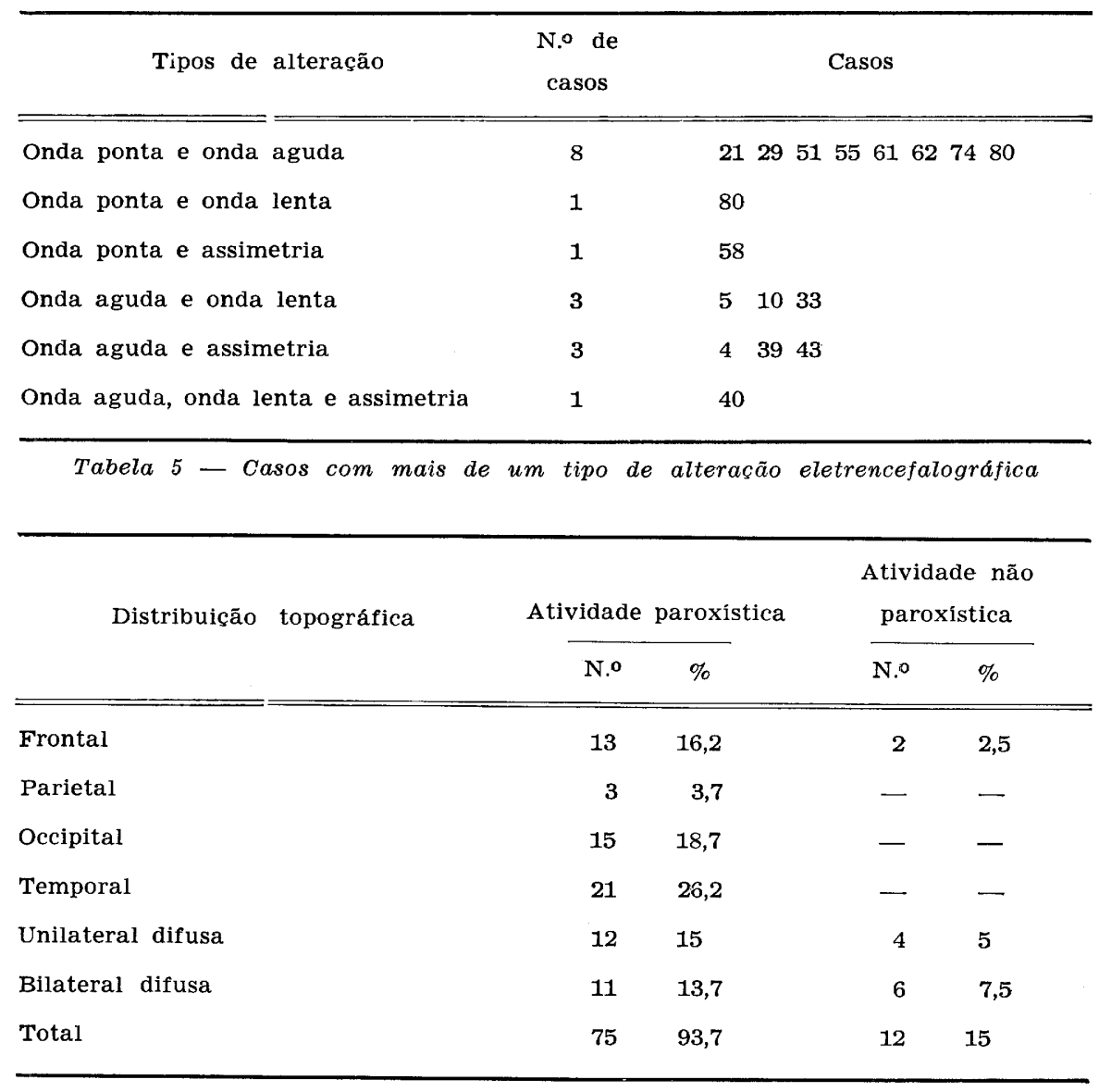

Tabela 6 - Distribuição topográfica da atividade paroxistica ou não

\begin{tabular}{|c|c|c|c|c|c|c|}
\hline \multirow[b]{2}{*}{ EEG } & \multicolumn{2}{|c|}{ Generalizada } & \multicolumn{2}{|c|}{ Parcial } & \multicolumn{2}{|c|}{ Unilateral } \\
\hline & N.o & $\%$ & N.o & $\%$ & N.o & $\%$ \\
\hline Focal & 22 & 27,4 & 6 & 7,5 & 12 & 15 \\
\hline Generalizada & 7 & 8,7 & - & - & - & - \\
\hline Alternante & 9 & 11,2 & 3 & 3,7 & 2 & 2,5 \\
\hline Normal & 10 & 12,5 & 2 & 2,5 & - & - \\
\hline Outros padrões & 2 & 2,5 & - & - & - & - \\
\hline Total & 50 & 62,3 & 11 & 13,7 & 14 & 17,5 \\
\hline
\end{tabular}

Tabela 7 - Correlacão entre os diferentes padrões bioelétricos e as crises clinicas 
A epilepsia é freqüente na infância, ocorrendo em 4 a $7 \%$ das crianças e sua incidência durante os primeiros dois anos é maior do que em qualquer outro período da vida ${ }^{5,22}$.

Apesar de serem numerosos os trabalhos sôbre os mecanismos fisiopatológicos da epilepsia, os estudos relacionados com a sistematização eletrencefalográfica dos diversos aspectos bioelétricos das crises convulsivas da infância são pouco freqüentes. A maioria das investigações se restringe a determinados aspectos do problema, abordando algumas das manifestações clínicas, não permitindo uma apreciação geral. Poucos são os trabalhos que examinam os dados obtidos especificamente mediante o EEG.

Esses fatos merecem ser salientados, pois a finalidade do presente estudo é avaliar, do ponto de vista qualitativo e quantitativo, o significado dos achados eletrencefalográficos paroxísticos de projeção focal ou difusa.

Os mecanismos responsáveis pela produção da atividade elétrica cerebral, com características epileptógenas, são ainda obscuros. O problema da determinação da origem dêsses vários padrões bioelétricos é de interêsse mais teórico, ao passo que a correta interpretação do significado, fisiológico ou patológico, de determinado potencial cerebral é de importância no campo de eletrencefalografia clínica.

$\mathrm{Na}$ análise dos fatôres envolvidos nos mecanismos de produção da atividade convulsiógena paroxística de nossos pacientes consideramos apenas nossos resultados eletrencefalográficos, comparados com a análise da literatura. Com êsse critério, foram analisadas inicialmente as características fisiopatológicas da atividade paroxística de projeção focal e representada pelas ondas aguda, lenta e em ponta; em seguida, estudamos a atividade paroxística de projeção difusa e bilateral representada pelo sincronismo bilateral secundário e pela atividade paroxística irregular, na forma e na freqüência.

Análise da atividade paroxística de projeção focal - Uma das características do foco convulsiógeno no EEG de crianças com menos de 12 meses de idade, é a variação, de momento a momento, de sua amplitude e duração. Estas modificações estão na dependência direta da idade e, portanto, da maturação cerebral; nestas circunstâncias o limiar convulsiógeno na criança é muito mais baixo que no adulto ${ }^{19}$.

Nossos resultados demonstraram que, durante o primeiro mês de vida, as descargas eletrencefalográficas são bem localizadas, muito embora sejam comuns as variações topográficas dos focos em um mesmo traçado (Fig. 1). Gastaut e Pinsard ${ }^{12}$ demonstraram que, do ponto de vista clínico, as manifestações convulsiógenas também são de tipo parcial, mesmo naqueles pacientes com patologia cerebral difusa.

Penfield e Jasper ${ }^{28}$ admitem que a atividade paroxistica focal tem incidência topográfica variável segundo a intensidade dos mecanismos responsáveis pela dispersão da atividade convulsiógena e representa, portanto, um 

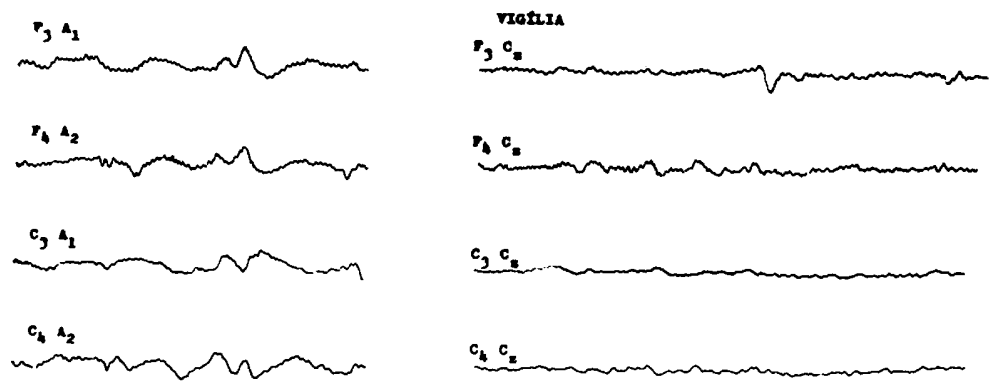

$\underbrace{c_{4} c_{2}}$
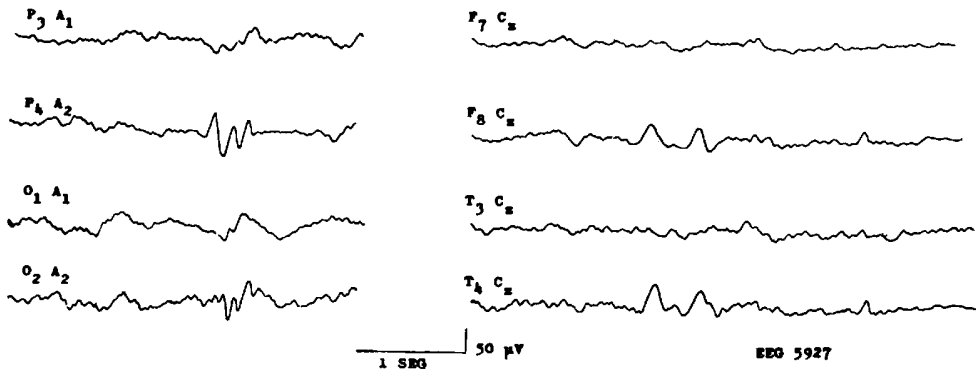

Fig. 1 - Atividade convulsiógena de projeção na região parieto-occipital direita e fronto-temporal direita, em paciєnte com 19 dias de idade.

fenômeno ligado à velocidade de condução da descarga originada usualmente em estruturas subcorticais. Nessas circunstâncias o foco representa como que um potencial evocado, de projeção em áreas cerebrais focais, porém variáveis.

Do ponto de vista topográfico, as regiões occipital e temporal, são áreas comuns para as projeções focais paroxísticas registradas no EEG de crianças até os três anos de idade ${ }^{3,14,40}$.

Em nosso material, focos convulsiógenos de projeção em áreas posteriores foram registrados em 18 pacientes $(22,4 \%)$, sendo de projeção na região occipital em 11 e, na região parietal, em dois (tabela 6).

A localização occipital de uma lesão focal constitui a maneira usual de reação do encéfalo da criança, embora êsses focos estejam na dependência de fatôres etiológicos diversos que, na maioria dos casos, são desprovidos de significado lesional topográfico.

A incidência do foco convulsiógeno de projeção no lobo temporal foi também um achado significativo no EEG de nossos pacientes e está de acôrdo com as investigações realizadas por Blanc e col. ${ }^{4}$. A atividade paroxística com características morfológicas de onda aguda predominou em áreas anteriores e médias do lobo temporal, enquanto que, nas áreas posteriores, foram registrados os focos com características de onda lenta, tipo delta ou teta. Apesar da alta incidência de anormalidades eletrencefalográficas nos pacientes com epilepsia temporal, o estudo eletrencefalográfico evidencia número ainda maior de anormalidades irritativas registradas com o eletrodo de profundidade e que 
não são observadas no EEG ${ }^{26}$. Os resultados obtidos com a eletrocorticografia demonstram a importância em se reconhecer os focos localizados na amígdala, corno de Ammon e circunvolução do hipocampo, pois as descargas originadas nestas estruturas rinencefálicas não são registradas usualmente nas derivações eletrencefalográficas de projeção nas regiões temporais ${ }^{25}$.

Teoria hastante atraente foi elaborada por Delange e col ${ }^{6,7}$ com o intúito de explicar a incidência das descargas focais para regióes específicas do lobo temporal. Admitem êstes autores que, para haver essa especificidade na distribuição topográfica das descargas epiléticas, o sistema de sincronização, ligado ao tálamo e à substância reticular do tronco cerebral, favorece um processo progressivo de inibição das manifestações corticais de projeção difusa. Nessas condições, as descargas originadas em níveis subcorticais e com tendência a se difundirem bilateralmente, passam a sofrer inibição direta das estruturas diencefálicas e, assim, exibem grande tendência a se manifestarem com característica focal, de projeção predominante nos lobos temporais. Foram as investigações estereotáxicas de Bancaud e col. ${ }^{2}$ que demonstraram a importância da organização funcional dessas estruturas cerabrais (diencéfalo, corno de Ammon, amigdala) no contrôle da modalidade de propagação das descargas elétricas de projeção focal em áreas temporais, e registradas principalmente no EEG realizado durante o sono.

Em outras palavras, a atividade convulsiógena de projeção na região temporal apresenta tendência a modificar as suas características morfológicas conforme a fase do sono o que, aliás, torna difícil muitas vêzes reconhecer o seu significado patológico; por outro lado, os focos de projeção na região frontal permanecem estáveis nas diversas fases do sono, constituindo um fator significativo de maturação cerebral ${ }^{36}$. Êsses eventos, registrados no EEG de crianças até 4 anos de idade, estão na dependência direta da mielinização encefálica, que se processa sucessivamente e que tem influência na morfologia do foco. Assim, a ação facilitadora de alguns fatôres que concorrem para baixar o limiar convulsiógeno, como é o caso da hipertermia, da predisposição genética e dos distúrbios metabólicos, favorece o aparecimento de crise com características clínicas relacionadas às condições de maturação cerebral ${ }^{12}$. Nessas circunstâncias, a presença de anormalidades paroxísticas pode indicar apenas imaturidade neuronal e só deve ser considerada no diagnóstico da epilepsia quando demonstrada em associação a outros paroxísmos bioelétricos patológicos evidenciados no registro eletrencefalográfico, pois, é a partir dos 4 anos de idade, que as diversas fases do sono adquirem características eletrencefalográficas mais precisas e que a reatividade aos estímulos sensoriais é mais estável, tornando o EEG semelhante ao do adulto 6, 21 .

Já os focos de projeção na região frontal apresentam incidência menor quando comparados com os focos de projeção em áreas posteriores e evidenciam, nas diversas fases do sono, maior estabilidade em sua morfologia quando comparados com os focos de projeção temporal; êsse fato é significativo de um processo de evolução das estruturas cerebrais ${ }^{26}$. 
Análise da atividade paroxistica de projeção difusa - A análise da atividade convulsiógena de projeção difusa e bilateral freqüentemente evidencia, ao lado dos padrões clássicos de $3 \mathrm{~Hz}$, outra forma de atividade bioelétrica na forma e na freqüência 20 .

De acôrdo com nosso material, essas alterações são constituídas, do ponto de vista eletrencefalográfico, por uma atividade desorganizada, caracterizada por surtos assíncronos de ondas irregulares, com elevada amplitude, mesclada com rítmo rápido. Ocasionalmente parecem ser focais e, logo em seguida, são registradas difusamente, mas nunca ritmadas. Essse tipo de anormalidade foi descrito, em 1952, por Gibbs e Gibbs ${ }^{15}$ com o nome de hipsarritmia e é usualmente registrado em crianças com história clínica sugestiva de espasmo em flexão.

Samson-Dollfus e col. ${ }^{37}$ demonstraram que essas alterações eletrencefalográficas típicas da hipsarritmia são melhor registradas durante o EEG de vigília ou no sono leve. Durante o sono profundo, o traçado adquire uma aparência de quase periodicidade.

Hoje em dia está perfeitamente demonstrada a importância das estruturas subcorticais nos mecanismos de transmissão e propagação da descarga epileptógena de projeção bilateral. A sensibilidade dessas estruturas varia em função de sua reação à intensidade dos estímulos e à velocidade de propagação da descarga. A face mesial do lobo temporal, amígdala e hipocampo, possuem baixo limiar na propagação da descarga epileptógena, enquanto que o núcleo caudado, globo pálido e tálamo apresentam limiar elevado quando estimulados, evidenciando, assim, tendências a propagar a descarga para áreas mais ou menos localizadas ${ }^{1,45}$.

Como regra geral, uma atividade elétrica com baixo limiar pós-descarga estimulando fibras de conexão, isto é, de projeção difusa, oferece uma condição ideal para a generalização de uma crise uni ou bilateral, enquanto que uma atividade com elevado limiar pós-descarga tende a bloquear qualquer estímulo nêle originado e a sua manifestação clínica será caracterizada apenas pelos sintomas restritos àquela região cerebral ativada ${ }^{8}$. Nessas circunstâncias, o hipocampo e a amígdala, com baixo limiar, evidenciam propriedades anatômicas e funcionais suficientes para disseminarem uma descarga epilética, enquanto que os gânglios da base e o tálamo, com elevado limiar, inibem a propagação de uma descarga convulsiógena.

Penfield e Jasper ${ }^{28}$ admitem que, nessas circunstâncias, a atividade convulsiógena de projeção difusa e bilateral pode ser verificada também em pacientes com áreas epileptógenas extensas e de localização cortical.

Em geral, quanto mais profundo estiver localizado o foco lesional, maior será a possibilidade da sua generalização sôbre o traçado, principalmente durante o sono, quando o EEG pode evidenciar a presença de duas ou mais alterações paroxísticas de projeção difusa e bilateral (Fig. 2). Os mecanismos que regem a propagação dessas descargas parecem estar ligados à presença de fibras facilitadoras e inibidoras que podem ser ativados seletivamente ${ }^{10,30,32}$. 


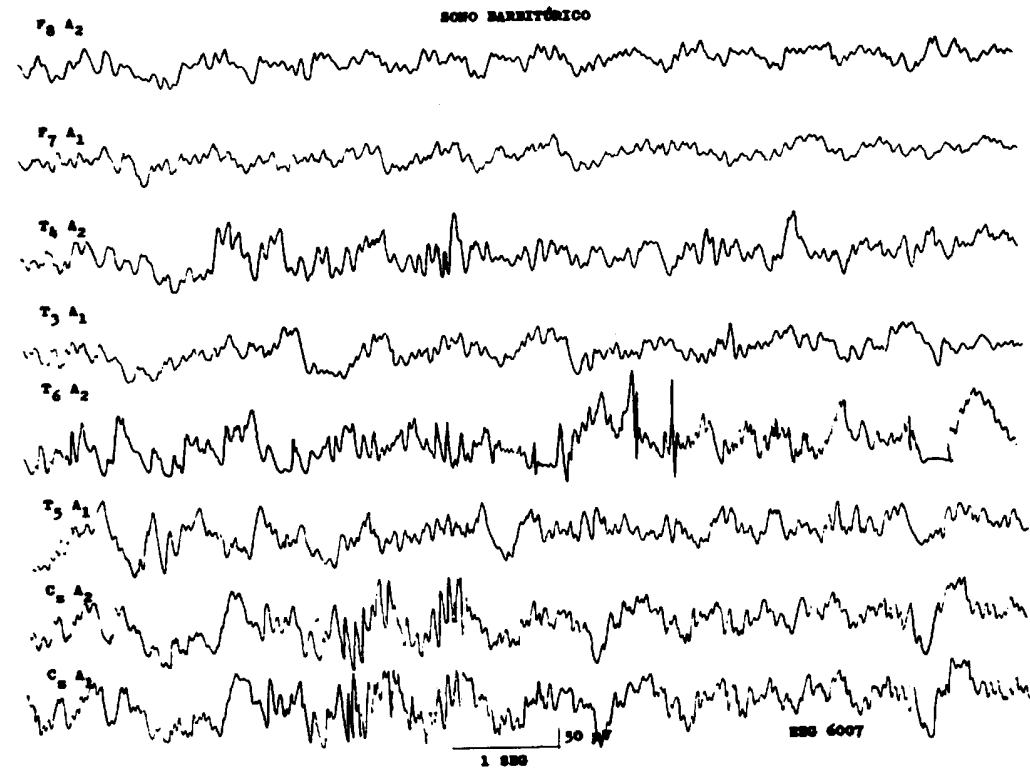

Fig. 2 - Onda ponta de projeção na região temporal posterior direita e onda aguda de projecão temporal média direita.

A origem focal paı a essas descargas deve ser levada em consideração, principalmente porque suas características eletrencefalográficas podem resultar do bombardeamento de lesões situadas no córtex e com projeção predominante para o tronco cerebral ${ }^{41}$. Até há algum tempo as investigações demonstravam que a propagação da atividade convulsiógena era relacionada ùnicamente com estruturas ligadas ao córtex cerebral, apenas algumas referências sendo feitas à participação dos centros subcorticais.

Atualmente, a importância dessas estruturas subcorticais nos mecanismos de propagação da atividade convulsiógena, de origem cortical, tem sido motivo de vários tipos de investigações. Trabalhos realizados em macacos demonstram que a participação de centros subcorticais na propagação de uma descarga originada em foco cortical passa a adquirir papel de relêvo se a área com a lesão cortical é extirpada após sensibilização prévia das estruturas subcorticais tendo participação apenas secundária se o córtex com o foco não sofre ablação ${ }^{9}$. Portanto, após a extirpação de área convulsiógena cortical, o impulso epileptógeno passa a ser originado nas estruturas subcorticais prèviamente sensibilizadas, seguindo um trajeto constituído pelas conexões da face mesial do lobo temporal contralateral com o hipocampo homolateral e complexo amigdalóide. Essas estruturas já "facilitadas" prèviamente pelo constante bombardeamento de impulsos originados em áreas corticais contralaterais, fàcılmente difundem a descarga, tanto para áreas homolaterais como contralaterais. Foram as investigações de Sloan e Jasper ${ }^{39}$, em 1950, e Tukel e Jasper 44, em 1952, que demonstraram a importância exer- 
cida pelo giro cingulado na difusão da atividade elétrica dos hemisférios cerebrais em animais sedados. As respostas obtidas por estimulação do giro cingulado eram mediadas e profundamente modificadas pelos núcleos talâmicos ântero-mediais e, possìvelmente, pela formação reticular talâmica ${ }^{32}$. Um foco cortical unilateral também pode originar descargas bilaterais e síncronas mediante estimulação de estruturas subcorticais, donde o têrmo "sincronismo bilateral secundário" em oposição a "sincronismo bilateral primário", característico do P.M. As duas formas de sincronismo podem ser diferenciadas, do ponto de vista eletrencefalográfico, pelas características morfológicas da descarga.

Êsse sincronismo bilateral de origem lesional focal não foi observado em estimulações de outras áreas, além do giro cingulado ${ }^{31}$. Entretanto, lesões irritativas na região da massa intermediária duplicam a intensidade do sincronismo bilateral, quando comparado com o sincronismo obtido de lesão cingular unilateral ${ }^{32}$.

Mais recentemente, trabalhos experimentais demonstraram que, no sistema nervoso central de epiléticos crônicos, a propagação da descarga pode se processar através de vias intranucleares mais ou menos complexas ${ }^{45}$. A compreensão dos fenômenos relacionados com a disseminação da descarga de uma crise focal está na dependência do conhecimento dos mecanismos físiopatológicos que podem ocorrer por ocasião da propagação de uma descarga epilética: 1) a descarga pode se propagar para área homóloga contralateral que é, então, estimulada; 2) a descarga se propaga para o tálamo homolateral que não responde à estimulação; 3) a descarga pode estimular o córtex temporal que, por sua vez, estimula a amígdala e hipocampo homolaterais e, em seguida, as estruturas temporais, hipotálamo e putamen do lado oposto; 4) a descarga pode estimular diretamente o putamen que pode, ou não, descarregar para o tálamo e tronco cerebral; 5) a descarga pode estimular os tratos córtico-bulbar e córtico-pontino e, assim, atingir os núcleos do tronco cerebral que, por sua vez, transmitem a descarga para o cerebelo e, através dêle, ao pedúnculo cerebral superior.

Nessas circunstâncias uma descarga focal pode se propagar para áreas do córtex homo oi contralateral e evidenciar, do ponto de vista eletrencefalográfico, padrões constituídos, na maioria das vêzes, por ondas delta de 2 a $3 \mathrm{~Hz}$ e com elevada amplitude, de projeção difusa. Outras vêzes essas alterações são evidenciadas em projeções difusas e bilaterais, mescladas com rítmos rápidos de $10 \mathrm{~Hz}$, acarretando um aspecto peculiar aos padrões ponta-onda lenta $23,24,35,38,43$.

\section{RESUMO E CONCLUSOES}

Foram estudadas, do ponto de vista eletrencefalográfico, 80 crianças epiléticas com a finalidade de investigar os aspectos qualitativos e quantitativos dos elementos patológicos paroxísticos.

Os resultados demonstraram que: 1) as manifestações paroxísticas focais apareceram de modo dominante nas projeções têmporo-occipitais; 2) o 
sono contribuiu para a identificação de duas ou mais alterações simultâneas nos traçados eletrencefalográficos; 3) a atividade paroxística de projeção difusa ou focal, evidencia distribuição topográfica independente do tipo de crise clínice; 4) as características bioelétricas da atividade paroxística não revelam diferenças significativas quando analisadas nos diferentes tipos de crises clínicas; 5) o início focal das crises generalizadas pode ser demonstrado pelo EF.G, mas êstes focos são em geral inconsistentes e mutantes; 6) o caráter inconstante das manifestações focais, a ausência de descargas generalizadas síncronas e o caráter variável do fenômeno convulsivo clínico, são conseqüè̀ncias da imaturidade cerebral.

\section{SUMMARY AND CONCLUSIONS}

\section{Paroxysmal electroencephalographic activity in the first year of life of epileptic patients}

The elestroencephalographic findings in 80 children with convulsive disorders occurring during the first year of life were analysed in order to investigate the qualitative and quantitative aspects of paroxistic patterns.

The following resuts were obtained: 1) the focal discharges were most commonly found over temporooccipital leads; 2) multiple focal discharges were more frequent recorded during sleep tracings; 3 ) there was no positive correlation between the site of the focus in EEG and concomitant clinical signs; 4) there was no positive correlation between bilateral or focal paroxystic activity and the types of seizures; 5) the initially focal course of a generalized seizure can be recorded on EEG, but the foci are inconstants and transients; 6) the inconstancy of focal discharges, the absence of bilateral paroxysmal pattein and the variability of convulsive phenomenon are related to the cerebral imaturity.

\section{REF E R E N C A S}

1. BANCAUD, J.; BLOCH, V. \& PAILLARD, J. - Contribuition à l'étude des potentiels évoqués chez l'homme au niveau du vertex. Rev. neurol. (Paris) $89: 399,1953$.

2. BANCAUD, J.; TALAIRACH, J.; BORDAS-FERRER, M.; AUBER, J. L. \& MARCHAND, H. - Les accès épileptiques au cours du sommeil de nuit (étude stéreo-électroencéphalographique). Electroenceph. clin. Neurophysiol. Suppl. 2: $255,1965$.

3. BLANC, C.; REBUFAT, M. \& DREYFUS-BRISAC, C. - Le problème des localisations occipitales chez l'enfant. Rev. neurol. (Paris) 101:287, 1959.

4. BLANC, C.; NEYRAUT, M. T. \& DREYFUS-BRISAC, C. - Note sur les localisations temporales chez l'enfant. Rev. neurol. (Paris) 103:256, 1960

5. BRIDGE, E. M. - Epilepsy and Convulsive Disorders in Children. McGrawHill, London, 1949. 
6. Delange, M.; CASTAN, Ph.; CADilhaC, J. \& PASSOUANT, P. - Etude EEG des divers stades du sommeil de nuit chez l'enfant; considérations sur le stade IV ou d'activité onirique. Rev. neurol. (Paris) 105:176, 1961.

7. DElange, P.; CASTAN, Ph.; CADIlHaC, J. \& PASSOUANT, P. - Etude du sommeil de nuit au cours d'épilepsies centrencéphaliques et temporales. Rev. neurol. (Paris) 106:106, 1962.

8. ELUL, R. - Regional differences in hippocampus of cat: projection of dorsal and ventral hippocampus. Electroenceph. clin. Neurophysiol. 16:489, 1964.

9. ERICKSON, T. C. - Spread of epileptic discharge; experimental study of afterdischarge induced by electrical stimulation of cerebral cortex. Arch. Neurol. Psychiat. (Chicago) 43:429, 1940.

10. FISCH, A. M.; OLIVEIRA, C. \& FERNANDES, I. - Contribuição ao estudo da hipsarritmia. Arq. Neuro-Psiquiat. (São Paulo) 24:15, 1966.

11. GASTAUT, H. et. al. - A proposed international classification of epileptic seizures. Epilepsia (Amsterdam) 5:297, 1964.

12. GASTAUT, H. \& PINSARD, N. - The electroencephalogram in children suffering from cerebral seizures. In Second Advanced Course in Electroencephalography. Vienna Academy of Medicine, Vienna, 1965.

13. GIBBS, F. A.; GIBBS, E. L. \& LENNOX, W. G. - Electroencephalographic response to overventilation and its relation to age. J. Pediat. (St. Louis) 23:497, 1943.

14. GIBBS, E. L.; GILLEN, H. W. \& GIBBS, F. A. - Disappearance and migration of epileptic foci in childhood. Amer. J. Dis. Child. 88:596, 1954.

15. GIBBS, F. A. \& GIBBS, E. L. - Atlas of Electroencephalography; Methodology and Controls. ed. 2. Addison-Wesley, Cambridge, 1959.

16. GIBBS, E. L.; RICH, H. L.; FOIS, A. \& GIBBS, F. A. - Electroencephalographic study of mentally retarded person. Amer. J. ment. Deficiency 65:236, 1960.

17. GIBBS, F. A. \& GIBBS, E. L. - Atlas of Electroencephalography: Neurological and Psychiatric Disorders. Addison-Wesley, Cambridge, 1964.

18. HESS Jr., R. - Sleep and sleep disturbances in the electroencephalogram. In A.kert, K.; Bally, C. \& Schadé, J. P.: Sleep Mechanisms. Elsevier, Amsterdam, 1965.

19. HILL, D. - Epilepsy: clinical aspects. In Hill, D. \& Parr, G.: Electroencephalography: a Symposium on its Various Aspects. MacDonald, London, 1963.

20. KILOH, L. G. \& OSSELTON, J. W. - Clinical Electroencephalography. Butterworths, London, 1961.

21. LARRY, G. C. - EEG of children with behavior disorders. In Second Advanced Course in Electroencephalography. Vienna Academy of Medicine, Vienna, 1965.

22. LIVINGSTON, S. - The Diagnosis and Treatment of Convulsive Disorders in Children. Charles C. Thomas, Springfield (Illinois), 1954.

23. LUNDERVOLD, A. - A prognostic evaluation of clinical, electroencephalographic and roentgenographic findings in children with epilepsy. Epilepsia (Amsterdam) $5: 33.1964$. 
24. NUFFIELD, E. J. A. - Electro-clinical correlactions in childhood epilepsy. Epilepsia (Amsterdam) 2:178, 1961.

25. PAGNI, C. A.; MASPES, P. E. \& CASSINARI, V. - Etude stéreo-électroencéphalographique du lobe temporal dans sept cas d'épilepsie psychmotrice avec foyer EEG temporal. Rev. neurol. (Paris) 108:98, 1963.

26. PALESTINI, M.; PISANO, M.; ROSADINI, G. \& ROSSI, G. F. — Excitability cycle of the visual cortex during sleep and wakefulness. Electroenceph. clin. Neurophysiol. 19:276, 1965.

27. PATERSON, D. - Convulsions (including epilepsy) in childhood. Arch. Dis. Childhood 26:441, 1951.

28. PENFIELD, W. \& JASPER, H. - Epilepsy and the Functional Anatomy of the Human Brain. Little Brown, Boston, 1954.

29. PETERSEN, I.; OLOFSSON, O.; HAGNE, I. \& SELLDẼN, U. - EEG of selected healthy children. Electroenceph. clin. Neurophysiol. 19:613, 1965.

30. POND, D. A. - The development of normal rhythms. In Hill, D. \& Parr, G.: Electroencephalography: a Symposium on its Various Aspects. MacDonald, London, 1963.

31. RALSTON, B. L. \& AJMONE-MARSAN, C. - Thalamic control of certain normal and abnormal cortical rhythms. Electroenceph. clin. Neurophysiol. 8:559, 1956.

32. RALSTON, B. L. - Cingulate epilepsy and secondary bilateral synchrony. Electroenceph. clin. Neurophysiol. 13:591, 1961.

33. RIBEIRO, R. M. - Achados eletrencefalográficos em pacientes com coriorretinite e reação de Sabin-Feldman positiva. Tese, Faculdade Medicina de Ribeirão Prêto, 1963.

34. RIBEIRO, R. M. - Achados eletrencefalográficos em crianças com crises convulsivas nos primeiros 12 meses de idade. Arq. Neuro-Psiquiat. (São Paulo) $24: 185,1966$.

35. RICCI, G. B. \& SCARINCI, A. - Clinical correlations and evolution in epileptics: EEG foci in children. Electroenceph. clin. Neurophysiol. 15:919, 1963.

36. SAMSON, D.; DELANGE-WALTER, M. \& MISES, J. - L'électroenphalogramme de l'enfant. Rev. neurol. (Paris) 108:138, 1963.

37. SAMSON-DOLLFUS, D.; FORTHOMME, J. \& CAPRON, E. - EEG of the human infant during sleep and wakefulness during the first year of life; normal patterns and their maturational changes; abnormal patterns and their prognostic significance. In Kellaway, P. \& Petersén, I.: Neurological and Electroencephalographic Correlative Studies in Infancy. Grune \& Stratton, New York, 1964.

38. SCOTT, J. S. \& KELLAWAY, P. - Epilepsy of focal origin in childhood. Med. clin. N. Amer. 3:415, 1958.

39. SLOAN, N. \& JASPER, H. H. - Studies of the regulatory functions of the anterior limbic cortex. Electroenceph. clin. Neurophysiol, 2:317, 1950.

40. SMITH, J. M. \& KELLAWAY, P. - The natural history and clinical correlates of occipital foci in children. In Kellaway, P. \& Petersén, I.: Neurological and Electroencephalographic Correlative Studies in Infancy. Grune \& Stratton, New York, 1964. 
188 ARQ. NEURO-PSIQUIAT. (SÃO PAULO) VOL. 27, N.9 3, SETEMBRO, 1969

41. STEWART, L. F. \& DREIFUSS, F. E. - "Centrencephalic" seizures discharges in focal hemispheral lesions. Arch. Neurol. (Chicago) 17:60, 1967.

42. STORM VAN LEEUWEN, W. et al. - Proposal for an EEG terminology by the committee of the International Federation for Electroencephalography and Clinical Neurophysiology. Electroenceph. clin. Neurophysiol. 20:293, 1966.

43. TROJABORG, W. - Focal spike discharges in children: a longitudinal study. Acta pediat. Scand. Supl. 168, 1966.

44. TUKEL, K. \& JASPER, H. H. - The electroencephalogram in parasagittal lesions. Electroenceph. clin. Neurophysiol. 4:481, 1952.

45. WALKER, A. E. \& UDVARHELYI, G. B. - Dissemination of acute focal seizure in the monkey from subcortical foci. Arch. Neurol. (Chicago) 12:357, 1965.

Departamento de Neurologia - Faculdade de Medicina de Ribeirão Prêto - Ribeirão Prêto. SP - Brasil. 